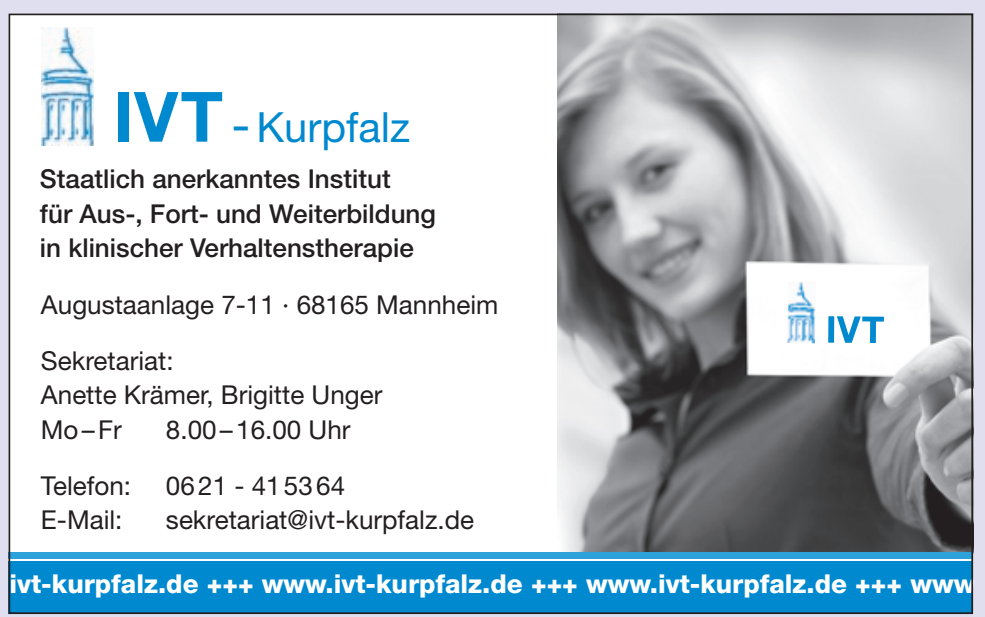

Institut für Fort- und Weiterbildung

in klinischer Verhaltenstherapie e. V.

Staatlich anerkanntes Ausbildungsinstitut

Weiterbildungen

$\rightarrow$ Schematherapie-Woche (ST 1 - ST 3) vom 06. bis 10.11 .2017

$\rightarrow$ Schematherapie-Kinder/Jugendliche (WS 3) am 22./23.09.2017

$\rightarrow$ Zusatzweiterbildung Kinder/Jugendliche (Fachkunde) Infotag: 20.09.2017, Start: 24./25.02.2018

Fortbildungstage

$\rightarrow$ Suizidrisiko bei Kindern und Jugendlichen am 21.10.2017

$\rightarrow$ Metakognitive Therapie bei Kindern und Jugendlichen am 18.11.2017

$\rightarrow$ Strukturelle Dissoziation am Beispiel der Dissoziativen Identitätsstörung am 24./25.11.2017

Bewerbungen/Anmeldungen ab sofort möglich.

Informationen und weitere Fortbildungsangebote:

IFKV • Kurbrunnenstr. 21 a • 67098 Bad Dürkheim • Tel. 06322 94828-0,

Fax: 06322 94828-29 • E-mail: info@ifkv.de • Internet: www.ifkv.de
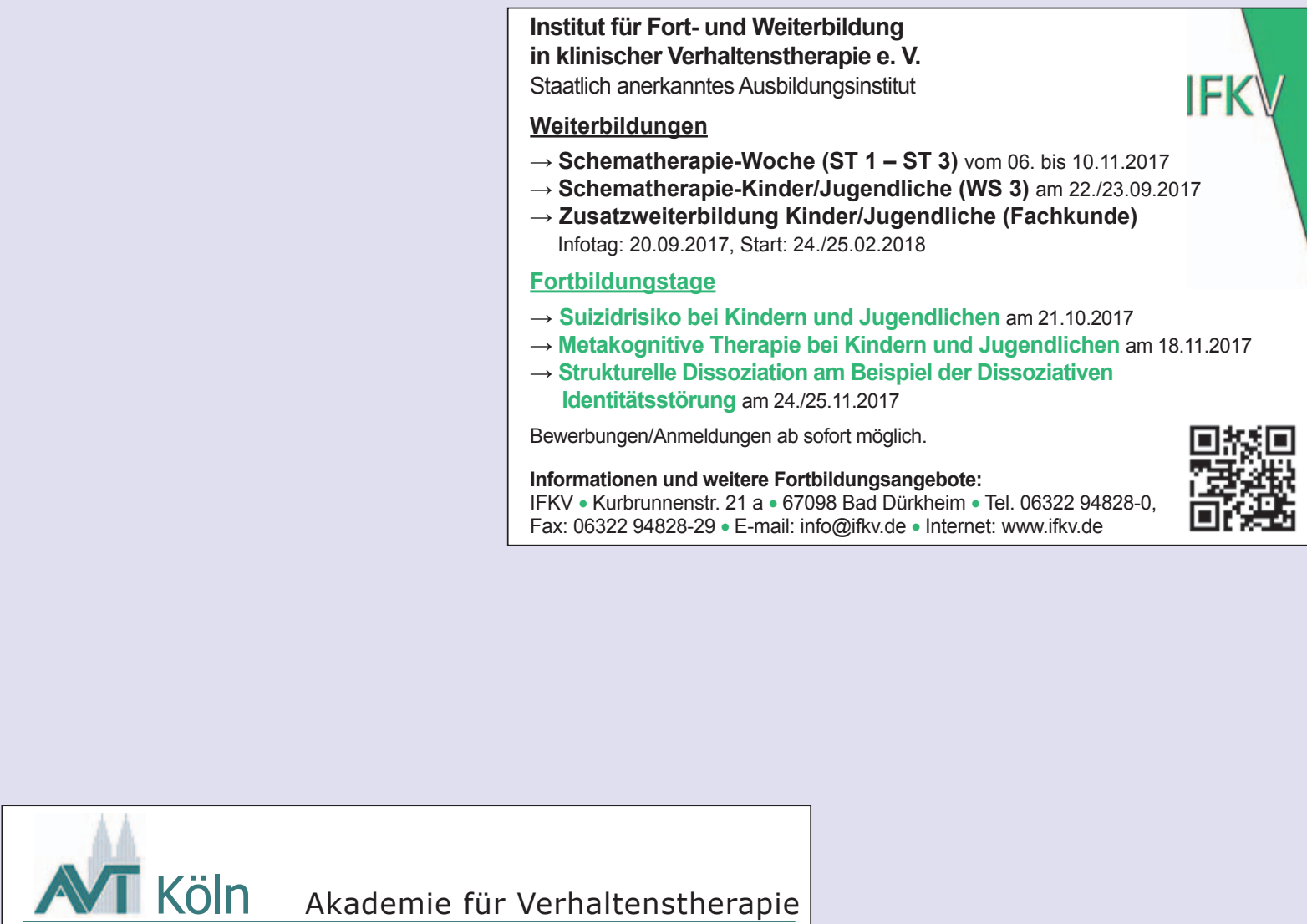

Akademie für Verhaltenstherapie

Staatlich anerkanntes Ausbildungsinstitut

Wir bieten:

O 3- und 5-jährige Ausbildung zur / zum Psychologischen Psychotherapeutin/en

- Ausbildungsbeginn dreimal jährlich -

$O$ ärztliche Weiterbildung in Verhaltenstherapie

$O$ Fortbildungsveranstaltungen

für Approbierte Psychotherapeuten

AVT GmbH • Venloer Straße 47-53 • 50672 Köln • www.avt-koeln.org Tel.: 0221 - 2585640 • Fax: 0221 - 25856420 • info@avt-koeln.org 
EMDR-Instifut Deutschland

der autorisierte Partner des Instituts von

Francine Shapiro PhD, USA

\section{Zertifizierte EMDR-Ausbildunc}

für Ärztl. und Psychol. Psychotherapeutlnnen und appr. KJP. Praxisbezogene Vermittlung unter Anleitung in Kleingruppen.

Gut erreichbare Seminarorte in Deutschland,

Österreich und Schweiz.

Info und Anmeldung:

www.emdr.de ·info@emdr.de·Tel.+49 - (0) 2204 - 25866

Staatlich anerkanntes Ausbildungsinstitut

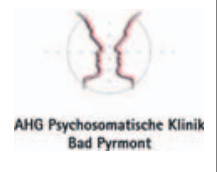

angegliedert an die AHG Psychosomatische Klinik Bad Pyrmont

Wir bieten:

- 3-jährige Vollzeitausbildung zum/r Psychologischen Psychotherapeuten/in

3-jährige Tätigkeit auf entsprechend vergüteten Stellen als

«Postgraduierte Psychologen» in der Klinik

- Sämtliche Ausbildungsbausteine werden in unserer Klinik bzw. in

unserem Ausbildungsinstitut durchgeführt.

- Die Kosten der Ausbildung tragen sich durch zusätzliche finanzielle

Unterstützung der Klinik und durch Einnahmen in der Ausbildungsambulanz.

- Ausbildungsbeginn jederzeit möglich

Information:

FIKV, Bombergallee 11, 31812 Bad Pyrmont

Tel. 05281 / 606763 oder 619620

sekretariat@fikv.de; www.fikv.de

\section{Verhaltenstherapie

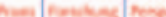

Hier könnte Ihr Fort- und Weiterbildungsangebot stehen!

Nutzen Sie Verhaltenstherapie zur effektiven

Kommunikation mit Ihrer Zielgruppe!

Informationen und Angebote bei

S. KARGER Verlag $\mathrm{GmbH}$

Ellen Zimmermann

Tel. +49761 45207-17

e.zimmermann@karger.com 